\title{
Contribution of peripheral neuropathy to poor bone health in the feet of people with type 2 diabetes mellitus
}

\author{
Joel Willem Johan Lasschuit ${ }^{1,2,3}$ - Jerry Richard Greenfield ${ }^{1,2,3} \cdot$ Katherine Thuy Trang Tonks $s^{1,2,3,4}$
}

Received: 22 June 2021 / Accepted: 13 September 2021 / Published online: 26 September 2021

(c) Springer-Verlag Italia S.r.l., part of Springer Nature 2021

\begin{abstract}
Aims To evaluate the impact of peripheral neuropathy on bone health in people with type 2 diabetes mellitus (T2DM). Methods Participants with T2DM were grouped according to the presence of peripheral neuropathy as assessed by vibration perception threshold (VPT). Recruitment ensured groups were balanced for age, sex and body mass index (BMI). Bone health was measured by calcaneal quantitative ultrasound (QUS) and compared between groups. Calcaneal QUS parameters were correlated across the cohort with VPT and other prespecified variables.

Results Thirty-four participants (17 per group) were included with mean age $68 \pm 12$ years, $47 \%$ male, with median BMI 29.9 (IQR $26.9-32.7) \mathrm{kg} / \mathrm{m}^{2}$. The peripheral neuropathy group had significantly lower mean Stiffness Index ( $87 \pm 12$ versus $101 \pm 16, p=0.01)$, Speed of Sound $(1542 \pm 28$ versus $1574 \pm 34 \mathrm{~m} / \mathrm{s}, p<0.01)$, and a trend towards lower Broadband Ultrasound Attenuation $(113 \pm 10$ versus $120 \pm 12 \mathrm{~dB} / \mathrm{MHz}, p=0.07)$. Pedal bone health asymmetry was not a significant feature in those with peripheral neuropathy. All calcaneal QUS parameters correlated negatively with VPT, although significance of the relationship with Broadband Ultrasound Attenuation was nullified if controlled for diabetes duration or time on insulin. Broadband Ultrasound Attenuation showed independent negative correlation with diabetes duration.

Conclusions People with T2DM and peripheral neuropathy have poorer bone health as measured by calcaneal QUS than those without peripheral neuropathy, independent of age, sex and BMI.
\end{abstract}

Keywords Type 2 diabetes mellitus $\cdot$ Peripheral neuropathy $\cdot$ Bone health $\cdot$ Bone density $\cdot$ Fracture $\cdot$ Calcaneal quantitative ultrasound

\section{Introduction}

Poor bone health is increasingly recognised as a complication of type 2 diabetes mellitus (T2DM). The pathophysiological mechanisms are complex and contentious, with obesity and insulin resistance observed to have varied effects on

Managed By Antonio Secchi .

Joel Willem Johan Lasschuit

j.lasschuit@garvan.org.au

1 Department of Endocrinology and Diabetes, St Vincent's Hospital, Sydney, NSW, Australia

2 Healthy Ageing, Garvan Institute of Medical Research, Sydney, NSW, Australia

3 Faculty of Medicine, University of New South Wales, Sydney, NSW, Australia

4 School of Medicine, University of Notre Dame, Sydney, NSW, Australia bone, and oxidative stress and microvascular disease likely detrimental to bone health in the later stages of T2DM $[1,2]$. A recent meta-analysis found that T2DM is associated with significantly increased risk of hip and vertebral fractures [3]. Of particular interest, the greatest elevation of fracture risk in T2DM was seen at the foot, where relative risk was $37 \%$ higher than comparator populations without T2DM, raising the potential involvement of peripheral neuropathy in fracture predisposition. Reported predictors of fracture are generally surrogates of disease severity, such as older age, longer diabetes duration, higher glycosylated haemoglobin and insulin use. Therefore, unravelling the contribution of peripheral neuropathy to fracture risk is problematic in large epidemiological studies.

There are potential adverse effects of peripheral neuropathy both extrinsic and intrinsic to bone that may contribute to increased fracture risk. Extrinsically, falls risk is increased in T2DM [4] and proprioceptive loss and gait disorders secondary to peripheral neuropathy are thought to 
partly mediate this risk [5]. However, the literature suggests increase risk of fracture in T2DM is independent of falls. Intrinsically, the neuropeptides substance $\mathrm{P}$ and calcitonin gene-related peptide (CGRP) regulate bone turnover in vitro $[6,7]$. In rodent models, selective injury to peripheral neurons is associated with reduced bone density, strength and unfavourable microarchitecture [8].

A particular conundrum is presented by Charcot neuropathic osteoarthropathy (or Charcot foot). Typically the condition presents unilaterally, an observation not explained by traditional Neurovascular and Neurotraumatic theories of the pathophysiology [9]. While the neuropathic foot is clearly susceptible to trauma, the majority of individuals who develop Charcot foot do not recall a distinct injury [10]. Focal osteopenia can be demonstrated in the acute Charcot foot [11]. However, it is unclear whether a baseline asymmetry in bone health precedes onset of the condition giving rise to its typically unilateral presentation.

If bone is intrinsically altered by peripheral neuropathy, one might anticipate measurable changes clinically. Dualenergy X-ray absorptiometry (DXA) is considered the gold standard for measurement of central bone density; however, it is not clinically validated for measuring pedal bone. In contrast, calcaneal quantitative ultrasound (QUS) is specifically designed to measure bone health at the heel and is portable, affordable and easy to use [12]. Utility for fracture risk prediction in T2DM is comparable to bone densitometry, and therefore this tool has potential application in screening [13]. Vibration perception threshold (VPT) is widely recommended in screening for clinically relevant diabetes-related peripheral neuropathy $[14,15]$. Assuming clinically measurable deleterious effects on bone requires well-established denervation, subtle pre-clinical disease detectable using more invasive techniques (e.g. nerve conduction studies, tissue biopsy) may be less relevant.

This study aimed to evaluate bone health using calcaneal QUS in people with T2DM and peripheral neuropathy, as compared to a T2DM group without peripheral neuropathy. Of importance, participant selection was designed to reduce confounders commonly unaccounted for in previous studies.

\section{Methods}

Participants were ambulant adults with T2DM attending St Vincent's Hospital Sydney Diabetes Clinics between 2018 and 2020. Exclusion criteria included previous osteotomy or surgical fixation involving the foot, previous major lower limb amputation, non-weight bearing for a period of 2 months or greater during the past year, any antiresorptive therapy during the past 12 months and/or a greater than 5-year cumulative exposure, pre-existing diagnosis of peripheral arterial disease and/or impalpable pedal pulses, heel ulceration at the site of QUS probe and/or greater than Texas Classification 1A foot ulceration, current foot infection and/or fracture, and acute or chronic Charcot neuropathic osteoarthropathy.

Two participant groups were recruited, those without and those with peripheral neuropathy. Clinical records were screened according to the eligibility criteria and previous documentation of peripheral neuropathy. The latter was used to guide recruitment, ensuring similarity in age, sex and body mass index (BMI) between groups. All potential participants were provided with details of the study, and, if interested, they were recruited. Signed informed consent was obtained. Final group allocation was based on the average of bilateral VPT with peripheral neuropathy defined at or above $25 \mathrm{~V}$. The threshold of $25 \mathrm{~V}$ is commonly used in clinical studies to indicate high risk of foot ulceration [16], and a VPT above this threshold is abnormal irrespective of age [17]. The sample size was set at 20 participants per group based on previous similar studies [18]; however, ultimately the COVID-19 pandemic hindered recruitment.

Data collection was cross sectional, with all assessments and measurements performed in a single visit by the same investigator (JL). First, clinical data were collected including demographics, diabetes history, fracture history, age of menopause (if relevant), current medications, previous exposure to antiresorptive, corticosteroid or hormone therapies, calcium and vitamin D supplementation, smoking status, alcohol consumption and physical activity (assessed by International Physical Activity Questionnaire) [19]. Second, anthropometry and foot sensation were assessed, including 4-point monofilament and VPT using methods outlined by Boulton et al. (2008) [20]. VPT was measured by calibrated Biothesiometer, Bio-Medical Instrument Co (Ohio, US). Blood was collected for calcium, magnesium, phosphate, parathyroid hormone level, 25-hydroxyvitamin D and glycosylated haemoglobin.

Bilateral QUS was performed using the Lunar Achilles EXP II, GE Healthcare (Wisconsin, US). This device reports Speed of Sound (SOS) and Broadband Ultrasound Attenuation (BUA), and amalgamates both measures in computing the Stiffness Index (SI). Each foot was measured twice by the same investigator (JL) with repositioning.

\section{Statistical analysis}

Paired measurements were used to assess reliability and precision. Reliability was analysed by intraclass correlation coefficients (ICC) with 95\% confidence intervals (CI) using a two-way mixed effects model with absolute agreement. Precision was analysed using coefficients of variation (CV) [21], which were then standardised to the variance of measurements within the study population [22]. The 95\% CI for CVs were determined by bootstrapping. 
Bilateral QUS measurements were averaged for each participant to allow comparison of SOS, BUA and SI between groups. Outliers were excluded from subsequent analysis if the SI was more than 1.5 times the interquartile range above the upper quartile. Remaining values were normally distributed and therefore group means compared using independent-samples t-tests.

The absolute difference between feet for each participant and QUS parameter was also calculated. These values were non-normally distributed, and therefore medians were reported and compared using Mann-Whitney U tests. Association of QUS parameters with VPT was evaluated using Pearson correlation across both groups combined. Previous meta-analyses have associated age, BMI, diabetes duration, $\mathrm{HbA} 1 \mathrm{c}$ and time on insulin with bone health $[3,23]$. Therefore, partial correlation was used to examine the robustness of the association between QUS parameters and VPT, controlling for aforementioned covariates in turn. In addition, while not the primary aim of this study, the same variables were correlated with QUS parameters as an exploratory analysis.

Statistical analysis was conducted using SPSS Statistics for Macintosh, version 25 (IBM Corp, Armonk, NY, USA). Bootstrapping was performed on R Studio for Macintosh, version 1.4.1106 (R Studio, Boston, MA, USA). Figures were created on GraphPad Prism for Macintosh, version 9.1.0 (GraphPad Software, San Diego, CA, USA). Ethics approval for the study was obtained from the St Vincent's Hospital Sydney Human Research Ethics Committee (reference number HREC/17/SVH/116) and the study was conducted in accordance with the Declaration of Helsinki.

\section{Results}

Screening of consecutive clinical records identified 52 potential participants. Following formal eligibility assessment, one potential participant was excluded due to the presence of internal ankle fixation, 9 declined participation, and 7 did not return contact. Thus, 35 individuals were studied: 17 without peripheral neuropathy and 18 with peripheral neuropathy. One outlier in the peripheral neuropathy group was excluded from further analysis (SI 1.7 times the interquartile range above the upper quartile), representing a male participant who had used testosterone for 10 years.

Participant characteristics are summarised in Table 1. Those with peripheral neuropathy were more likely to be using insulin ( $82 \%$ versus $41 \%, p=0.01)$ and have other microvascular complications ( $77 \%$ versus $35 \%, p=0.02$ ).

Intraobserver reliability was excellent for all QUS parameters in this cohort. The intraclass correlation coefficients were 0.98 (95\% CI 0.96-0.99) for SOS, 0.96 (95\% CI 0.92-0.98) for BUA and 0.995 (95\% CI 0.99-1.00) for SI.
Intraobserver precision was well within ranges previously reported [12]. Coefficients of variation for SOS, BUA and SI were $0.3 \%, 2.0 \%$ and $1.3 \%$ respectively. Corresponding standardised coefficients of variation were $3.2 \%, 4.8 \%$ and $1.8 \%$.

As illustrated in Fig. 1, bone parameters measured by QUS were poorer in the peripheral neuropathy group. In particular, SI and SOS were significantly lower, and a trend towards lower BUA was observed. Asymmetry between feet was not statistically significantly greater in those with as compared to those without peripheral neuropathy. Median difference between feet for SI was $3.0(1.0-7.5)$ in those without neuropathy and $7.0(2.3-11.5)$ in those with neuropathy $(p=0.15)$. The respective figures for SOS were 6.1 $(2.4-13.9) \mathrm{m} / \mathrm{s}$ versus $11.5(2.2-19.9) \mathrm{m} / \mathrm{s}(p=0.63)$ and for BUA $3.7(1.8-8.3) \mathrm{dB} / \mathrm{MHz}$ versus $6.0(2.2-9.1) \mathrm{dB} / \mathrm{MHz}$ $(p=0.43)$.

Significant correlations between all QUS parameters and VPT were seen across the cohort. Pearson correlation coefficients $(r)$ were $-0.54(p=0.001)$ for SOS, -0.40 for BUA $(p=0.02)$ and -0.52 for SI $(p=0.002)$. Figure 2 shows the correlation of SI with VPT. Significant correlation of VPT with SOS and SI remained with partial correlation analyses to control for confounders. In contrast, the relationship between VPT and BUA was no longer significant if controlled for diabetes duration $(r=-0.33, p=0.07)$ or time on insulin $(r=-0.43, p=0.05)$. There were no statistically significant correlations between SOS or SI and age, BMI, diabetes duration, HbA1c or time on insulin. Broadband Ultrasound Attenuation correlated significantly with diabetes duration $(r=-0.37, p=0.03)$ but not with the other variables.

\section{Discussion}

In this study, we demonstrated that people with T2DM and peripheral neuropathy have poorer bone health than those without peripheral neuropathy, as measured by calcaneal QUS. As groups were similar with respect to age, sex and BMI, the association appears to be independent of these factors. When VPT was considered as a continuum, there was a significant negative correlation with all calcaneal QUS parameters, primarily driven by SOS. Pedal bone health asymmetry was not more pronounced in those with as compared to those without peripheral neuropathy.

Previous studies evaluating the impact of peripheral neuropathy on bone health in T2DM have shown incongruous results [24]. On the one hand, significant association of neuropathy with fracture risk has been demonstrated in people with T2DM over the age of $50[25,26]$. Of note, these studies were retrospective and neuropathy was identified as a predictor in multivariate analyses. On the other hand, several 
Table 1 Participant characteristics grouped according to the presence of peripheral neuropathy

\begin{tabular}{|c|c|c|c|c|c|}
\hline \multirow{2}{*}{$\overline{\text { Demographics }}$} & \multicolumn{2}{|c|}{ No neuropathy $(n=17)$} & \multicolumn{2}{|c|}{ Neuropathy $^{\#}(n=17)$} & \multirow[t]{2}{*}{$p$-value } \\
\hline & & & & & \\
\hline Age (years) & 68 & $( \pm 12)$ & 67 & $( \pm 13)$ & 0.84 \\
\hline Gender (men) & 8 & $(47 \%)$ & 8 & $(47 \%)$ & 1.00 \\
\hline Ethnicity (Caucasian) & 14 & $(82 \%)$ & 15 & $(88 \%)$ & 0.63 \\
\hline \multicolumn{6}{|l|}{ Diabetes } \\
\hline Duration (years) & 18 & $( \pm 9)$ & 21 & $( \pm 12)$ & 0.40 \\
\hline Insulin use & 7 & $(41 \%)$ & 14 & $(82 \%)$ & $0.01 *$ \\
\hline Time using insulin (years) & 6 & $(3-15)$ & 7 & $(3-14)$ & 0.82 \\
\hline Duration of neuropathy symptoms (years) & - & & 5 & $(2-10)$ & - \\
\hline Other microvascular complication $/ \mathrm{s}$ & 6 & $(35 \%)$ & 13 & $(77 \%)$ & $0.02 *$ \\
\hline Any macrovascular complication/s & 3 & $(18 \%)$ & 8 & $(47 \%)$ & 0.07 \\
\hline \multicolumn{6}{|l|}{ Bone } \\
\hline Previous low trauma fracture & 3 & $(18 \%)$ & 4 & $(24 \%)$ & 0.67 \\
\hline Time post-menopause (years, for women) & 21 & $( \pm 12)$ & 18 & $( \pm 10)$ & 0.58 \\
\hline Previous antiresorptive use & 0 & & 0 & & - \\
\hline Calcium supplement & 4 & $(24 \%)$ & 1 & $(6 \%)$ & 0.15 \\
\hline Vitamin D supplement & 9 & $(53 \%)$ & 10 & $(59 \%)$ & 0.73 \\
\hline Family history of osteoporosis & 1 & $(6 \%)$ & 0 & & - \\
\hline \multicolumn{6}{|l|}{ Social } \\
\hline Smoking (past/current) & 8 & $(47 \%)$ & 7 & $(41 \%)$ & 0.73 \\
\hline Alcohol ( $\geq 1 \mathrm{SD} /$ day) & 5 & $(29 \%)$ & 5 & $(29 \%)$ & 1.00 \\
\hline Physical activity (MET-min/week) & 1210 & $(960-1750)$ & 990 & $(500-1390)$ & 0.22 \\
\hline \multicolumn{6}{|l|}{ Clinical } \\
\hline Body mass index $\left(\mathrm{g} / \mathrm{cm}^{2}\right)$ & 29.3 & $(24.8-32.0)$ & 30.5 & $(28.8-32.5)$ & 0.39 \\
\hline Waist circumference $(\mathrm{cm})$ & 106 & $( \pm 14)$ & 108 & $( \pm 12)$ & 0.72 \\
\hline Monofilament (4/4 bilaterally) & 12 & $(71 \%)$ & 2 & $(12 \%)$ & $<0.001 *$ \\
\hline Biothesiometry (V) & 16 & $(12-22)$ & 39 & $(31-44)$ & $<0.001$ *\# \\
\hline \multicolumn{6}{|l|}{ Biochemistry } \\
\hline HbA1c (\%) & 7.2 & $( \pm 1.2)$ & 7.7 & $( \pm 1.2)$ & 0.21 \\
\hline HbAlc (mmol/mol) & 55 & $( \pm 13)$ & 61 & $( \pm 13)$ & 0.21 \\
\hline Corrected calcium (mmol/L) & 2.37 & $( \pm 0.12)$ & 2.38 & $( \pm 0.13)$ & 0.74 \\
\hline 25-OH vitamin D (nmol/L) & 61 & $( \pm 16)$ & 60 & $( \pm 21)$ & 0.86 \\
\hline Parathyroid hormone (pmol/L) & 5.1 & $( \pm 2.4)$ & 4.5 & $( \pm 1.8)$ & 0.44 \\
\hline
\end{tabular}

* Statistically significant difference between groups at $p<0.05$

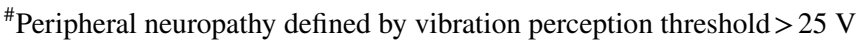

Parametric data mean (SD); nonparametric data median (IQR); other data $n(\%)$

$S D$ standard drink (10 g alcohol); MET metabolic equivalent of task studies have reported on bone outcomes using dual-energy X-ray absorptiometry (DXA), X-ray morphometry, QUS or peripheral quantitative computed tomography, with only two suggesting unfavourable outcomes in people with neuropathy $[27,28]$.

While the literature on bone health in type 1 diabetes mellitus (T1DM) is plentiful, studies dedicated to investigating bone outcomes in people with peripheral neuropathy and T2DM are less prevalent. Many have included individuals with both T1DM and T2DM. The pathophysiology of these conditions is clearly very distinct, as are the proposed deleterious effects of diabetes on bone health. For instance, the peak incidence of T1DM onset is in adolescence, which impacts on the accrual of peak bone mass [29]. Additionally, the deficiency of anabolic hormones, particularly insulin, insulin-like growth factor- 1 and amylin, is thought to contribute to poorer bone health in people with T1DM [30, 31].

In contrast, T2DM is a state of hyperinsulinaemia at onset of the disease and osteoblasts in T2DM may exert resistance to the effects of anabolic hormones [32,33]. Bone health is also likely to be adversely affected by advanced glycation end-products, increased marrow adiposity and diminished incretin effect [34-36]. As much of the existing evidence has arisen from large population cohorts and retrospective 
Fig. 1 Comparison of calcaneal quantitative ultrasound parameters in people without or with peripheral neuropathy
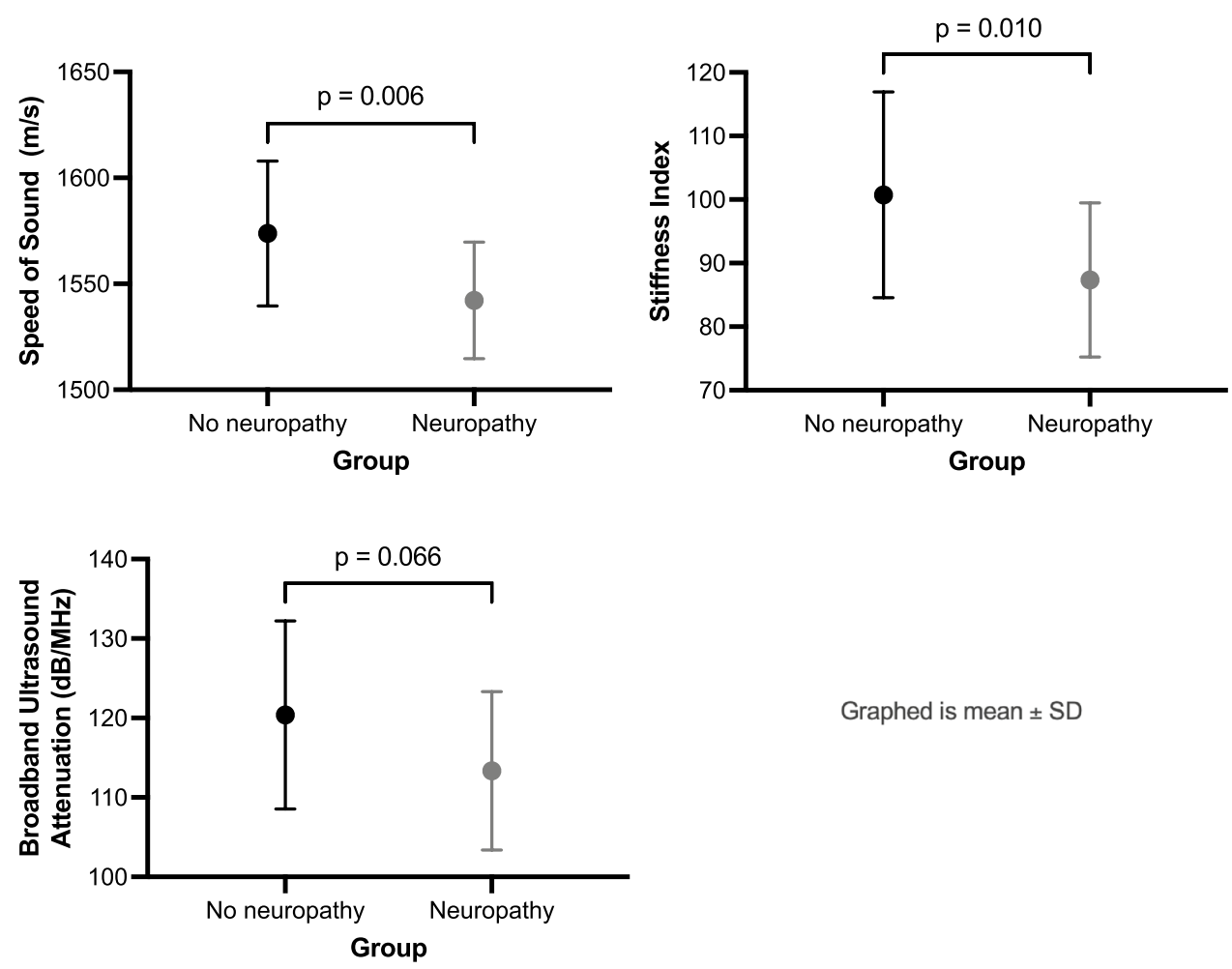

Group

Graphed is mean \pm SD

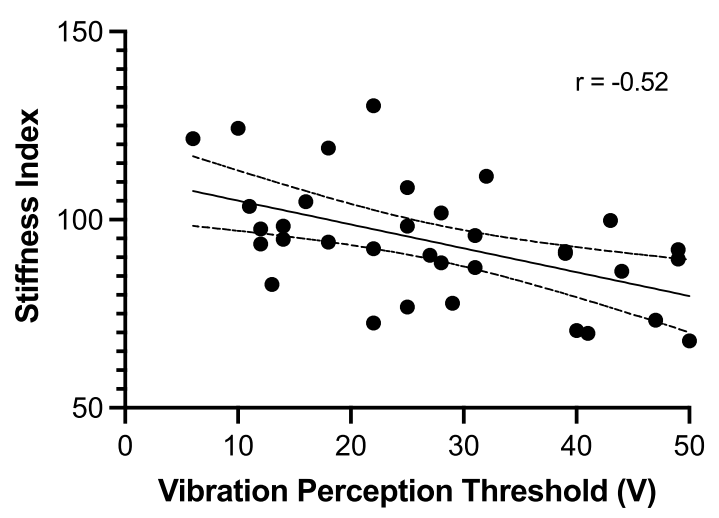

Fig. 2 Pearson correlation of Stiffness Index with vibration perception threshold across all participants

analyses, matching or adjusting for confounders is often not possible. For example, the presence of peripheral arterial disease, Charcot foot, vitamin D deficiency or previous antiresorptive use would be anticipated to have independent effects on bone.

Our study specifically recruited people with T2DM, and balanced groups for age, gender and BMI. Further, detailed eligibility criteria reduced many of the aforementioned confounders. Some characteristics did differ between the groups, in particular insulin use and coexisting microvascular complications. It is interesting that insulin use was higher in the peripheral neuropathy group despite similar diabetes duration and BMI. As with the prevalence of coexisting diabetes complications, this is likely to be a marker of disease severity and needs to be considered in interpreting our findings. Although not a significant finding in our study, physical activity as measured by the International Physical Activity Questionnaire was previously reported to be lower in those with peripheral neuropathy [37], which could influence bone health.

The key findings on calcaneal QUS were lower SOS and SI in the peripheral neuropathy group. SOS is thought to be more representative of bone density, given the speed of ultrasound transmission is impeded by the bone mineral content. In contrast, BUA theoretically incorporates assessment of bone quality or microarchitecture as the refraction of ultrasound reflects the degree of porosity. Our data therefore suggests the overall SI, which is an amalgamation of SOS and BUA, was primarily driven by lower bone density. Further, asymmetry was not a significant feature in those with peripheral neuropathy. This supports the hypothesis that osteopenia is a consequence of rather than a predisposing factor to acute Charcot foot [9]. In our experience, significant asymmetry on calcaneal QUS is primarily seen in acute Charcot foot and situations where preferential loading occurs, for example in elite athletes (unpublished data).

The Health ABC Study included 2200 individuals between 70 and 79 years of age, with approximately 20 per cent prevalence of diabetes mellitus [28]. Type of diabetes mellitus was not specified. Peripheral nerve function 
was assessed by monofilament, VPT and peroneal motor nerve conduction velocity and amplitude, with the results used to group participants in quartiles. Lower total hip bone mineral density using DXA and calcaneal BUA using QUS were associated with poorer nerve function. The interesting exception was VPT for calcaneal BUA, for which there was no significant association. Our study used VPT to define peripheral neuropathy and BUA was also not significantly different between groups.

By contrast, two prior studies combining people with T1DM and T2DM did not report a significant association between peripheral neuropathy and bone health using QUS. Piaggesi et al. (2002) using the same VPT cut-off of $25 \mathrm{~V}$ found SOS and BUA to be comparable in those with $(n=14)$ or without $(n=13)$ neuropathy, noting approximately $30 \%$ in each group had T1DM [38]. Urine deoxypyridinoline, a marker of bone resorption, was higher in the group with neuropathy. Chakrabarty et al. (2004) studied people at the time of diabetes diagnosis, with $23 \%$ prevalence of T1DM, and a vast age range from 11 to 70 years. Only BUA was measured, and no correlation was seen with peripheral neuropathy [39].

More recent studies dedicated to T2DM have used highresolution peripheral quantitative tomography (HR-pQCT) to assess bone quality. Microvascular disease was associated with higher cortical porosity, and lower cortical volumetric BMD and cortical thickness at the distal radius [40, 41], with a trend towards higher cortical porosity at the distal tibia [41]. The results are not directly comparable to the current study given the prevalence of peripheral neuropathy amongst those with microvascular disease was low or unspecified, and the definition of peripheral neuropathy varied.

The major strength of our study was the similarity between groups in terms of key determinants of bone health, specifically age, sex and BMI, and careful use of exclusion criteria to reduce confounders. The groups were well characterised with respect to both diabetes characteristics and peripheral neuropathy, as compared to retrospective population-based studies. A single investigator performed all measurements ensuring consistency, and very high QUS reliability and precision was proven. Given the absence of blinding, the relatively subjective assessment of VPT was recorded prior to calcaneal QUS measurement, which is objective. Power was limited by the relatively small number of participants, although the sample size per group was not dissimilar to previous comparable studies. The cross-sectional study design does not allow for exploration of causality. There was a potential for selection bias, although all eligible clinic attendees were approached within the limits of the study methodology. Finally, only post-menopausal women were recruited, primarily given that peripheral neuropathy as a time-dependent complication of T2DM is more common in this age group.
More studies are needed to investigate the impact of peripheral neuropathy on bone health in T2DM using carefully characterised cohorts. Assuming our findings are reproducible, several clinically relevant hypotheses are raised. Peripheral neuropathy may partly explain the higher risk of pedal fractures in T2DM as compared to the general population and also contribute to the complex pathophysiology that underlies Charcot foot. Bone health may simply be a marker of disease severity and detrimental effects of hyperglycaemia on bone. However, perhaps poor pedal bone health is a manifestation of microvascular ischaemic disease, denervation, hyperinsulinaemia and/or insulin resistance.

We have previously proposed that QUS may have utility in bone health screening [13]; however, this data now suggests one would need to simultaneously screen for peripheral neuropathy. Peripheral measures of bone health are less likely to be representative of general bone health when peripheral neuropathy is present. In this case, central DXA may be more relevant. While we have previously shown that QUS is predictive of any low trauma fracture in T2DM [13], specific relevance to foot fracture and Charcot foot prediction would require further evaluation. Of interest, if QUS could be used to identify those at greatest risk of Charcot foot prior to its onset, there may be opportunity for preventative action. Finally, it is unclear whether interventions commonly used in managing osteopenia reduce fracture risk in T2DM, particularly if peripheral neuropathy is contributing to bone health deterioration.

\section{Conclusions}

Peripheral neuropathy in T2DM seems to be associated with poorer bone health as measured by calcaneal QUS, independent of age, sex and BMI. Across the spectrum of VPT, there is a robust negative correlation with calcaneal QUS parameters, driven by SOS. Finally, the presence of peripheral neuropathy does not appear to lead to greater asymmetry in pedal bone health. Larger studies in clinically well-defined longitudinal cohorts are required to better understand the relationship between peripheral neuropathy in T2DM and bone health.

Acknowledgements J.L. was supported by an Australian Government Research Training Program Scholarship via the University of New South Wales, Sydney. The Sky Foundation kindly funded biochemical testing and facility costs.

Author contributions All authors contributed to the study conception and design. Material preparation, data collection and analysis were performed by Dr Joel Lasschuit. The first draft of the manuscript was written by Dr Joel Lasschuit, and all authors commented on previous versions of the manuscript. All authors read and approved the final manuscript. 
Funding J.L. was supported by an Australian Government Research Training Program Scholarship via the University of New South Wales, Sydney. The Sky Foundation kindly funded biochemical testing and facility costs.

\section{Declarations}

Conflict of interest The authors declare that they have no conflict of interest.

Data availability The datasets generated during and/or analysed during the current study are available from the corresponding author on reasonable request.

Ethics approval This study was performed in line with the principles of the Declaration of Helsinki. Approval was granted by the St Vincent's Hospital Sydney Human Research Ethics Committee (reference number HREC/17/SVH/116).

Consent to participate Informed consent was obtained from all individual participants included in the study.

Consent to publish Participants signed informed consent regarding publishing their data.

\section{References}

1. Shanbhogue VV, Hansen S, Frost M, Brixen K, Hermann AP (2017) Bone disease in diabetes: another manifestation of microvascular disease? Lancet Diabetes Endocrinol 5(10):827-838. https://doi.org/10.1016/S2213-8587(17)30134-1

2. Shanbhogue VV, Mitchell DM, Rosen CJ, Bouxsein ML (2016) Type 2 diabetes and the skeleton: new insights into sweet bones. Lancet Diabetes Endocrinol 4(2):159-173. https://doi.org/10. 1016/S2213-8587(15)00283-1

3. Moayeri A, Mohamadpour M, Mousavi SF, Shirzadpour E, Mohamadpour S, Amraei M (2017) Fracture risk in patients with type 2 diabetes mellitus and possible risk factors: a systematic review and meta-analysis. Ther Clin Risk Manag 13:455-468. https://doi.org/10.2147/TCRM.S131945

4. Yang Y, Hu X, Zhang Q, Zou R (2016) Diabetes mellitus and risk of falls in older adults: a systematic review and meta-analysis. Age Ageing 45(6):761-767. https://doi.org/10.1093/ageing/afw140

5. Mayne D, Stout NR, Aspray TJ (2010) Diabetes, falls and fractures. Age Ageing 39(5):522-525. https://doi.org/10.1093/ageing/ afq081

6. Wang L, Shi X, Zhao R, Halloran BP, Clark DJ, Jacobs CR et al (2010) Calcitonin-gene-related peptide stimulates stromal cell osteogenic differentiation and inhibits RANKL induced NFkappaB activation, osteoclastogenesis and bone resorption. Bone 46(5):1369-1379. https://doi.org/10.1016/j.bone.2009.11.029

7. Wang L, Zhao R, Shi X, Wei T, Halloran BP, Clark DJ et al (2009) Substance P stimulates bone marrow stromal cell osteogenic activity, osteoclast differentiation, and resorption activity in vitro. Bone 45(2):309-320. https://doi.org/10.1016/j.bone.2009.04.203

8. Offley SC, Guo TZ, Wei T, Clark JD, Vogel H, Lindsey DP et al (2005) Capsaicin-sensitive sensory neurons contribute to the maintenance of trabecular bone integrity. J Bone Miner Res 20(2):257-267. https://doi.org/10.1359/JBMR.041108

9. Jeffcoate WJ (2008) Charcot neuro-osteoarthropathy. Diabetes Metab Res Rev 24(Suppl 1):S62-S65. https://doi.org/10.1002/ dmrr.837
10. Schon LC, Easley ME, Weinfeld SB (1998) Charcot neuroarthropathy of the foot and ankle. Clin Orthop Relat Res 349:116-131

11. Jirkovska A, Kasalicky P, Boucek P, Hosova J, Skibova J (2001) Calcaneal ultrasonometry in patients with Charcot osteoarthropathy and its relationship with densitometry in the lumbar spine and femoral neck and with markers of bone turnover. Diabet Med. 18(6):495-500. https://doi.org/10.1046/j.1464-5491.2001.00511.x

12. Krieg MA, Barkmann R, Gonnelli S, Stewart A, Bauer DC, Del Rio BL et al (2008) Quantitative ultrasound in the management of osteoporosis: the 2007 ISCD Official Positions. J Clin Densitom 11(1):163-187. https://doi.org/10.1016/j.jocd.2007.12.011

13. Lasschuit JWJ, Center JR, Greenfield JR, Tonks KTT (2019) Comparison of calcaneal quantitative ultrasound and bone densitometry parameters as fracture risk predictors in type 2 diabetes mellitus. Diabet Med. https://doi.org/10.1111/dme.14183

14. American Diabetes A. (2021) 11. Microvascular Complications and Foot Care: Standards of Medical Care in Diabetes-2021. Diabetes Care 44(Suppl 1):S151-S67. https://doi.org/10.2337/ dc21-S011

15. Bus SA, Lavery LA, Monteiro-Soares M, Rasmussen A, Raspovic A, Sacco ICN et al (2020) Guidelines on the prevention of foot ulcers in persons with diabetes (IWGDF 2019 update). Diabetes Metab Res Rev 36(Suppl 1):e3269. https://doi.org/10.1002/dmrr. 3269

16. Garrow AP, Boulton AJ (2006) Vibration perception threshold-a valuable assessment of neural dysfunction in people with diabetes. Diabetes Metab Res Rev 22(5):411-419. https://doi.org/10.1002/ dmrr.657

17. Maffei L, Premrou V, Roldan P, Copetti M, Pellegrini F, Rossi MC et al (2014) Vibration perception threshold in the screening of sensorimotor distal symmetric polyneuropathy: the need of more accurate age-specific reference values. J Diabetes Sci Technol 8(3):621-622. https://doi.org/10.1177/1932296814527818

18. Barwick AL, de Jonge XA, Tessier JW, Ho A, Chuter VH (2014) The effect of diabetic neuropathy on foot bones: a systematic review and meta-analysis. Diabet Med 31(2):136-147. https:// doi.org/10.1111/dme.12347

19. IPAQ Group. International Physical Activity Questionnaire (IPAQ) 2002 [Available from: https://sites.google.com/site/theip aq/home]. Accessed 17 Jun 2017

20. Boulton AJ, Armstrong DG, Albert SF, Frykberg RG, Hellman R, Kirkman MS et al (2008) Comprehensive foot examination and risk assessment: a report of the task force of the foot care interest group of the American Diabetes Association, with endorsement by the American Association of Clinical Endocrinologists. Diabetes Care 31(8):1679-1685. https://doi.org/10.2337/dc08-9021

21. Gluer CC, Blake G, Lu Y, Blunt BA, Jergas M, Genant HK (1995) Accurate assessment of precision errors: how to measure the reproducibility of bone densitometry techniques. Osteoporos Int 5(4):262-270. https://doi.org/10.1007/BF01774016

22. Orgee JM, Foster H, McCloskey EV, Khan S, Coombes G, Kanis JA (1996) A precise method for the assessment of tibial ultrasound velocity. Osteoporos Int 6(1):1-7. https://doi.org/10.1007/BF016 26530

23. Ma L, Oei L, Jiang L, Estrada K, Chen H, Wang Z et al (2012) Association between bone mineral density and type 2 diabetes mellitus: a meta-analysis of observational studies. Eur J Epidemiol 27(5):319-332. https://doi.org/10.1007/s10654-012-9674-x

24. Beeve AT, Brazill JM, Scheller EL (2019) Peripheral Neuropathy as a Component of Skeletal Disease in Diabetes. Curr Osteoporos Rep 17(5):256-269. https://doi.org/10.1007/s11914-019-00528-8

25. Kim JH, Jung MH, Lee JM, Son HS, Cha BY, Chang SA (2012) Diabetic peripheral neuropathy is highly associated with nontraumatic fractures in Korean patients with type 2 diabetes mellitus. Clin Endocrinol (Oxf) 77(1):51-55. https://doi.org/10.1111/j. 1365-2265.2011.04222.x 
26. Lee RH, Sloane R, Pieper C, Lyles KW, Adler RA, Van Houtven $\mathrm{C}$ et al (2018) Clinical fractures among older men with diabetes are mediated by diabetic complications. J Clin Endocrinol Metab 103(1):281-287. https://doi.org/10.1210/jc.2017-01593

27. Cundy TF, Edmonds ME, Watkins PJ (1985) Osteopenia and metatarsal fractures in diabetic neuropathy. Diabet Med 2(6):461-464. https://doi.org/10.1111/j.1464-5491.1985.tb00683.x

28. Strotmeyer ES, Cauley JA, Schwartz AV, de Rekeneire N, Resnick HE, Zmuda JM et al (2006) Reduced peripheral nerve function is related to lower hip BMD and calcaneal QUS in older white and black adults: the Health, Aging, and Body Composition Study. J Bone Miner Res 21(11):1803-1810. https://doi.org/10.1359/jbmr. 060725

29. Liu EY, Wactawski-Wende J, Donahue RP, Dmochowski J, Hovey KM, Quattrin T (2003) Does low bone mineral density start in post-teenage years in women with type 1 diabetes? Diabetes Care. 26(8):2365-9. https://doi.org/10.2337/diacare.26.8.2365

30. Heald A, Stephens R, Gibson JM (2006) The insulin-like growth factor system and diabetes-an overview. Diabet Med 23(Suppl 1):19-24. https://doi.org/10.1111/j.1464-5491.2006.01833f.x

31. Horcajada-Molteni MN, Chanteranne B, Lebecque P, Davicco MJ, Coxam V, Young A et al (2001) Amylin and bone metabolism in streptozotocin-induced diabetic rats. J Bone Miner Res 16(5):958-965. https://doi.org/10.1359/jbmr.2001.16.5.958

32. Napoli N, Chandran M, Pierroz DD, Abrahamsen B, Schwartz AV, Ferrari SL et al (2017) Mechanisms of diabetes mellitus-induced bone fragility. Nat Rev Endocrinol 13(4):208-219. https://doi.org/ 10.1038/nrendo.2016.153

33. Tonks KT, White CP, Center JR, Samocha-Bonet D, Greenfield JR (2017) Bone Turnover Is Suppressed in Insulin Resistance, Independent of Adiposity. J Clin Endocrinol Metab 102(4):1112-1121. https://doi.org/10.1210/jc.2016-3282

34. Hein GE (2006) Glycation endproducts in osteoporosis-is there a pathophysiologic importance? Clin Chim Acta 371(1-2):32-36. https://doi.org/10.1016/j.cca.2006.03.017

35. Sheu Y, Schwartz A, Amati F, Goodpaster BH, Li X, Bauer D et al (2012) Bone marrow adiposity is elevated in older men with type 2 diabetes. Diabetes 61(Suppl 1):A367
36. Sanz C, Vazquez P, Blazquez C, Barrio PA, Alvarez Mdel M, Blazquez E (2010) Signaling and biological effects of glucagonlike peptide 1 on the differentiation of mesenchymal stem cells from human bone marrow. Am J Physiol Endocrinol Metab 298(3):E634-E643. https://doi.org/10.1152/ajpendo.00460.2009

37. Johnson NA, Barwick AL, Searle A, Spink MJ, Twigg SM, Chuter VH (2019) Self-reported physical activity in community-dwelling adults with diabetes and its association with diabetes complications. J Diabetes Complications 33(1):33-38. https://doi.org/10. 1016/j.jdiacomp.2018.10.017

38. Piaggesi A, Rizzo L, Golia F, Costi D, Baccetti F, Ciaccio S et al (2002) Biochemical and ultrasound tests for early diagnosis of active neuro-osteoarthropathy (NOA) of the diabetic foot. Diabetes Res Clin Pract 58(1):1-9. https://doi.org/10.1016/s01688227(02)00097-9

39. Chakrabarty N, Sarkar P, Pal SK, Banerjee R, Sarkar RN, Debnath NB (2004) A study of bone mineral density in diabetes mellitus in eastern India. J Indian Med Assoc 102(8):418

40. de Waard EAC, de Jong JJA, Koster A, Savelberg H, van Geel TA, Houben A et al (2018) The association between diabetes status, $\mathrm{HbA1c}$, diabetes duration, microvascular disease, and bone quality of the distal radius and tibia as measured with high-resolution peripheral quantitative computed tomography-The Maastricht Study. Osteoporos Int 29(12):2725-2738. https://doi.org/10.1007/ s00198-018-4678-3

41. Shanbhogue VV, Hansen S, Frost M, Jorgensen NR, Hermann AP, Henriksen JE et al (2016) Compromised cortical bone compartment in type 2 diabetes mellitus patients with microvascular disease. Eur J Endocrinol 174(2):115-124. https://doi.org/10.1530/ EJE-15-0860

Publisher's Note Springer Nature remains neutral with regard to jurisdictional claims in published maps and institutional affiliations. 